\title{
Controlling elements come of age
}

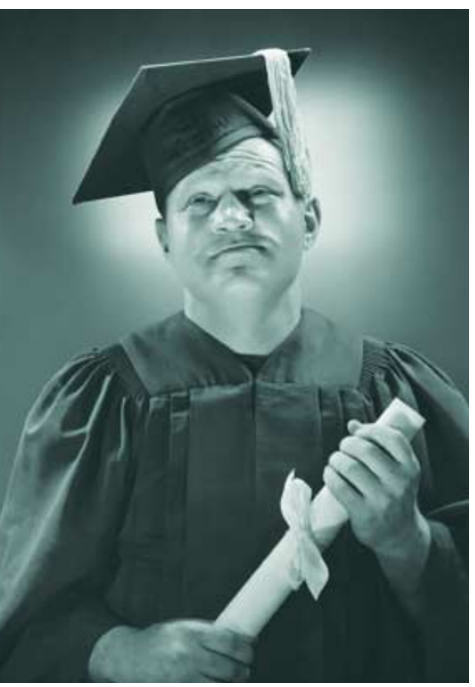

Until recently, we believed that it was the job of proteins to regulate gene expression. More recently it has become clear that RNA is another important player in this process. Using mouse oocytes and preimplantation embryos, Barbara Knowles and co-workers now show that retrotransposons have an important role in gene regulation - possibly by controlling chromatin states.

That mobile elements can affect gene expression is not news - it has been known for a long time that transposon-promoter sequences can induce endogenous gene expression by transcriptional read-through. As far back as the 1950s, when Barbara
McClintock first described transposons, she referred to them as controlling elements. What was previously unappreciated, however, was the scale on which this might occur.

The authors' discovery began with the analysis of cDNA libraries from mature mouse oocytes, 2-cell-stage embryos and blastocysts. They observed that two types of retrotransposons were strikingly abundant among transcripts in oocytes and in 2-cell-stage embryos. But as well as these transcripts, the authors found abundant chimeric transcripts, made up of host sequences spliced downstream of alternative 5 ' retroelement sequences.
Aligning these chimeric sequences to the mouse genome showed that all of them came from genes that harboured the transposable elements, either within the gene locus or upstream of it.

Wanting to look into the potential roles of these chimeric transcripts, the authors compared their expression dynamics with that of the corresponding wild-type gene. The fact that the chimeric transcripts disappear after the 8-cell stage indicated that they are maternal transcripts, and the authors suggest that the long terminal repeats of these retrotransposons might behave as oocyte-specific promoters. Although some ORFs of these chimeric transcripts do not differ from the wildtype transcripts, others do, and as the authors show that at least some are translated, it is therefore possible that they might encode novel, stage-specific functions.

\section{HUMAN GENETICS}

\section{No simple answer to complex disease}

Testing SNPs for association with disease provides the most promising way of getting to the bottom of complex human genetic disorders. But how to pick the SNPs that are most likely to be involved in disease is a controversial matter. One suggestion is that the molecular basis of monogenic Mendelian diseases - which are better understood can be used as a model: a theory that is put to the test in a recent study by Paul Thomas and Anish Kejariwal.

Mutations that underlie Mendelian diseases are generally single-nucleotide changes that have a strong effect on protein function, consistent with the fact that these diseases involve single mutations with strong phenotypic effects. Complex diseases, on the other hand, are caused by multiple mutations, implying that each mutation has a weak effect on its own. This has led to the suggestion that SNPs that are associated with complex disorders are more likely to have milder effects on protein function or to affect regulatory non-coding regions. However, several studies of complex disease have identified SNPs in coding regions as having causal roles, indicating that their molecular basis might be similar to Mendelian disorders after all.
To test these possibilities, the authors examined SNPs identified from previous studies as being associated with complex disease and looked at how likely they are to have severe effects on protein function. They did this by determining how conserved the amino acids affected are in related proteins from different species - highly conserved positions are likely to have essential roles in protein function. Positions affected in complex disease turn out to be far less conserved than for Mendelian disorders, with a distribution similar to genetic variation in healthy individuals. So, unlike Mendelian disorders, polymorphisms that underlie complex genetic disease probably have small effects on protein function.

The study also provides insights into the evolution of the two types of disease. The fact that SNPs that are associated with complex disease generally affect non-conserved positions indicates that they might have been subject to little selective pressure over long evolutionary periods, unlike those involved in Mendelian disorders, which are likely to have been strongly selected against. To examine more recent events, the authors compared nucleotide sequences of disease-associated genes with those of their mouse orthologues, and used the ratio of non-synonymous and synonymous single-nucleotide changes as a measure of selective pressure - the more non-synonymous changes there have been, the greater the positive selective pressure is likely to have been. Whereas genes affected in Mendelian disease showed a relatively low level of non-synonymous changes, the level was higher for those associated with complex disease, which in fact seem to have been subject to more recent positive selective pressure than for human genes in general.

So, it seems our knowledge of how mutations affect protein function in Mendelian diseases cannot generally be used to predict variants that are likely to be associated with complex genetic disorders. Perhaps the evolutionary insights from this study will prove more helpful, with evidence of recent selective pressure revealing the genes that are likely to be involved, if not the variants themselves.

Louisa Flintoft

\section{(2) References and links} ORIGINAL RESEARCH PAPER

Thomas, P. D. \& Kejariwal, A. Coding single-nucleotide polymorphisms associated with complex vs. Mendelian disease: evolutionary evidence for differences in molecular effects. Proc. Natl Acad. Sci. USA 101, 15398-15403 (2004)

WEB SITE

Paul Thomas's laboratory:

http://panther.appliedbiosystems.com 
Support for the functional importance of this retrotransposonmediated transcription comes from phylogenetic conservation - the positioning of some of the retrotransposons associated with chimeric transcripts is conserved between two distantly related strains of mice and, in some cases, between rat and mouse. Unfortunately, what their function is remains an open question. The authors propose that sequential activation and silencing of retrotransposons might underlie stage-specific, potentially RNAimediated, chromatin remodelling at specific genomic locations at early stages of development and during oogenesis - an attractive model that urgently needs further testing.

Magdalena Skipper

\section{(0) References and links} ORIGINAL RESEARCH PAPER Peaston, A. E., Evsikov, A. V. et al. Retrotransposons regulate host genes in mouse oocytes and preimplantation embryos. Dev. Cell 7, 597-606 (2004)

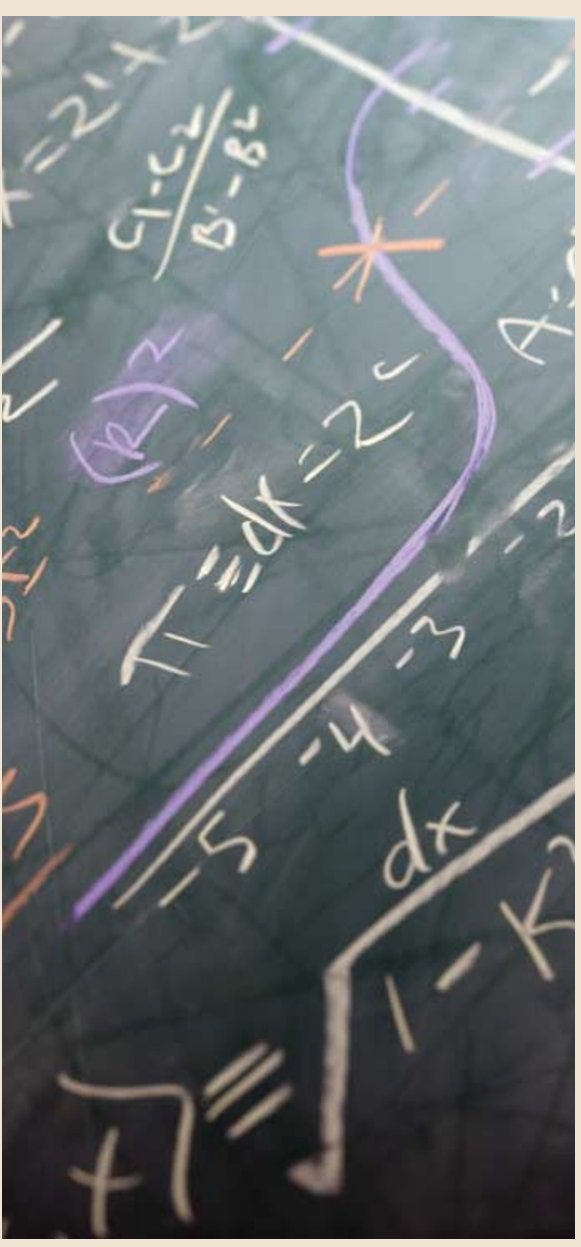

RNA INTERFERENCE

\section{Silencing the sceptics}

We've all heard of the promise of RNA interference (RNAi) as a potential approach to treating disease, but how close are we to delivering on this promise? Writing in Nature, Vornlocher and colleagues now take us a step closer to the goal of selectively modifying gene activity for a clinical benefit.

Vornlocher and colleagues have demonstrated the in vivo silencing of an endogenous gene that encodes a protein of therapeutic relevance, apolipoprotein $B$ (APOB), which is essential for the formation of lowdensity lipoprotein (LDL) and therefore implicated in a range of cardiovascular disorders. Although RNAi has been demonstrated in vivo before, previous models have either not targeted an endogenous gene or have been administered in a way that is not applicable to the human therapeutic setting. In this study, small interfering RNA (siRNA) molecules, which induce RNAi of genes with complementary sequences, were administered to mice by tail-vein injection, a route that can be readily translated to human patients.

Just as gene therapy and antisense approaches have been held back by problems of delivery, so too has RNAi. In the past, viral vectors have been used, among other delivery vehicles, to get siRNA to the target tissue. This study used chemical modifications to render the siRNA molecules more drug-like and stable within the body, and more likely to be taken up by cells. The conjugation of cholesterol to the $3^{\prime}$ end of siRNA molecules significantly improved their in vivo pharmacological properties, such as cellular delivery and half-life. Such improvements lend credence to the idea that further chemical modifications could improve the prospects of siRNA-based therapeutics.

To explore the in vivo effect of these $A p o B$ targeting, cholesterol-modified siRNAs, they were injected into mice that were fed a normal diet. Levels of the target mRNA were then measured in the liver and jejunum, key sites of APOB expression. The modified siRNA caused a significant reduction of $A p o B$ mRNA in both tissues, which was reflected as a diminution of APOB protein levels. Desirable effects were seen at the physiological level too: siRNA treatment resulted in a $25 \%$ reduction in high-density lipoprotein levels, and a $40 \%$ reduction in LDL levels.

A key concern with studies of RNAi is that the observed results might be caused by nonspecific 'offtarget' effects or the interferon response. Vornlocher and colleagues were able to eliminate this possibility, because two $A p o B$-specific siRNAs that target different regions of the $A p o B$ mRNA resulted in similar effects. Furthermore, control siRNA, although present in the liver and jejunum at levels comparable to

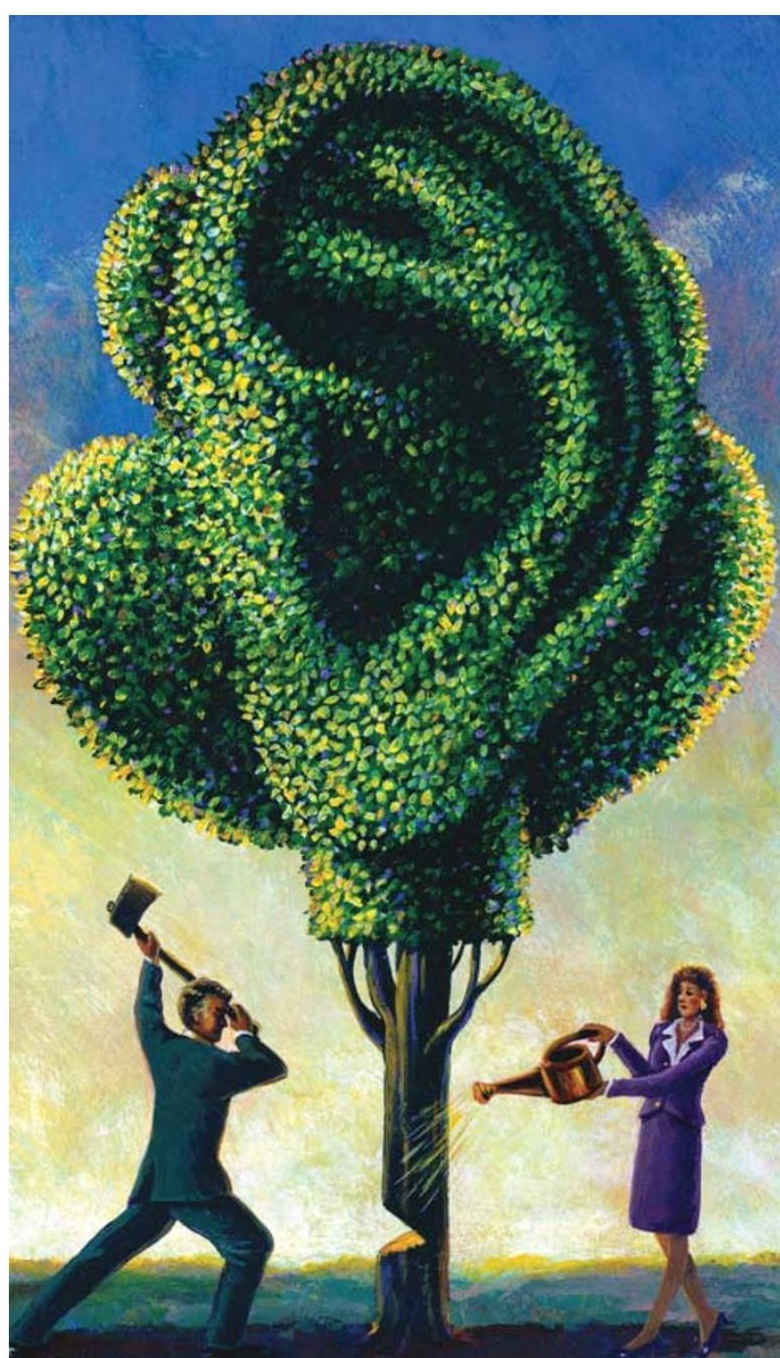

those of therapeutic siRNA, had no effect. The authors were also able to establish, through an analysis of APOB degradation products, that an RNAi mechanism of action was responsible for the experimental findings.

With the first demonstration of the in vivo silencing by siRNA of an endogenous gene through an RNAi mechanism - and using an administration route that could readily be applied to humans — in the bag, RNAi looks set to gain even further prominence as a research tool and potential therapeutic.

Daniel Jones

\section{0) References and links}

ORIGINAL RESEARCH PAPER Soutschek, J. et al. Therapeutic silencing of an endogenous gene by systemic administration of

modified siRNAs. Nature 11 November 2004 (doi:10.1038/nature) FURTHER READING Hall, J. Unravelling the general properties of siRNAs: strength in numbers and lessons from the past. Nature Rev. Genet. 5, 552-557 (2004) 